\title{
INSTRUMENTO DE COLETA DE DADOS PARA PACIENTES CRÍTICOS FUNDAMENTADO NO MODELO DAS NECESSIDADES HUMANAS BÁSICAS DE HORTA ${ }^{a}$
}

\author{
Rosaura Costa BORDINHÃO ${ }^{\mathrm{a}}$, Miriam de Abreu ALMEIDA ${ }^{\mathrm{b}}$
}

\section{RESUMO}

Trata-se de um estudo exploratório descritivo, com abordagem qualitativa, que teve como objetivo construir coletivamente um instrumento de coleta de dados para pacientes de Unidade de Terapia Intensiva (UTI), fundamentado na Teoria das Necessidades Humanas Básicas (NHB). A coleta de dados ocorreu por meio de Grupo Focal, com quatro enfermeiras de UTI e quatro residentes de Enfermagem da Residência Integrada em Saúde (RIS), em sete encontros, em 2009. As discussões produzidas em cada sessão foram analisadas conforme o preconizado por Horta. O instrumento ficou estruturado em sete grupos e 17 subgrupos de necessidades. Após testagem e sugestões das participantes, elaborou-se a versão final do instrumento e um manual de orientação para o seu preenchimento, conforme necessidade manifestada pelo grupo. Sugere-se a validação do instrumento e do manual e a inclusão do ensino do Processo de Enfermagem no programa de atividades da RIS.

Descritores: Processos de enfermagem. Coleta de dados. Unidades de Terapia Intensiva. Educação em saúde.

\section{RESUMEN}

Este es un estudio exploratorio con enfoque cualitativo con el objetivo de construir colectivamente un instrumento de recolección de datos para los pacientes en la Unidad de Cuidados Intensivos (UCI), fundamentado en la Teoría de las Necesidades Humanas Básicas (NHB). La recolección de datos fue a través de grupos focales con cuatro enfermeras de la UCI y cuatro residentes de la Residencia Integrada en Salud (RIS) en siete reuniones en 2009. Las discusiones producidas se analizaron según lo recomendado por Horta. El instrumento se dividió en siete grupos y 17 subgrupos de las necesidades. Después de las pruebas y sugerencias de los participantes, se elaboró la versión final del instrumento y un manual de orientación para su llenado, como la necesidad expresada por el grupo. Se sugiere validar el instrumento y el manual y la inclusión de la enseñanza del PE en el programa de actividades de la RIS.

Descriptores: Procesos de enfermería. Recolección datos. Unidades de Terapia Intensiva. Educación en salud. Título: Instrumento de recolección de datos para pacientes críticos fundamentada en la teoría de las necesidades humanas básicas.

\section{ABSTRACT}

This is an exploratory study based on qualitative approach that aimed to collectively construct an instrument to collect data for patients in the Intensive Care Unit (ICU), based on the Theory of Basic Human Needs (NHB). Data collection was through a focus group with four nurses from the ICU and four residents from the Nursing-Health Integrated Residency (RIS) program in seven meetings in 2009. The discussions produced in each session were analyzed as recommended by Horta. The instrument was divided into seven groups and 17 subgroups of needs. After testing and suggestions from participants, we elaborated the final version of the instrument and a guidance manual for completing it, according to the need expressed by the group. Validation of the instrument and the manual and inclusion of teaching of the nursing process in the RIS activity program are suggested.

Descriptors: Nursing process. Data collection. Intensive Care Units. Health education.

Title: Instrument to collect data for critical patients based on the theory of basic human needs.

a Artigo extraído da Dissertação de Mestrado apresentada em 2010 à Escola de Enfermagem da Universidade Federal do Rio Grande do Sul, intitulada: Processo de enfermagem em uma unidade de tratamento intensivo à luz da Teoria das necessidades humanas básicas.

b Mestre em Enfermagem, Enfermeira do Hospital Nossa Senhora da Conceição (HNSC), Porto Alegre, Rio Grande do Sul, Brasil.

c Doutora em Educação, Professora Associada da Escola de Enfermagem da Universidade Federal do Rio Grande do Sul (UFRGS), Porto Alegre, Rio Grande do Sul, Brasil. 


\section{INTRODUÇÃO}

O Processo de Enfermagem (PE) é um método sistematizador da assistência que embasa e direciona a prática profissional do enfermeiro. Ele foi introduzido no Brasil em meados da década de 1970, por Wanda Horta, que desenvolveu um modelo teórico baseado nas Necessidades $\mathrm{Hu}-$ manas Básicas (NHB) trabalhadas por Maslow na Teoria da Motivação Humana ${ }^{(1,2)}$. Essa teoria apóia-se e engloba três princípios gerais: a lei do equilíbrio (homeostase): todo o universo se mantém por processos de equilíbrio dinâmico entre os indivíduos; a lei da adaptação: os indivíduos procuram se manter em equilíbrio a partir da interação com o seu meio externo; e, a lei do holismo: o todo não é simplesmente a soma das partes, mas o conjunto delas ${ }^{(2)}$.

Além das NHB de Maslow, Horta adotou a denominação de João Mohana, em necessidades de nível psicobiológico, psicossocial e psicoespiritual ${ }^{(3)}$. As Necessidades Psicobiológicas são consideradas forças, instintos ou energias inconscientes que surgem sem planejamento, do nível psicobiológico do homem e se manifestam, por exemplo, na vontade de se banhar e repousar. As Necessidades Psicossociais são manifestações que ocorrem no indivíduo por meio de instintos do nível psicossocial, como a necessidade de comunicar-se, de viver em grupo e realizar trocas sociais. As Necessidades Psicoespirituais são aquelas por meio das quais o homem procura compreender o que vivencia de inexplicável cientificamente, transcendendo e ultrapassando as linhas que limitam sua experiência no mundo ${ }^{(4)}$.

Para tornar operacional o seu modelo, a autora propôs o Processo de Enfermagem em seis etapas inter-relacionadas, quais sejam: Histórico de Enfermagem, Diagnóstico de Enfermagem, Plano Assistencial, Prescrição de Enfermagem e Prognósticos de Enfermagem ${ }^{(3,4)}$. Com o avanço dos estudos relacionados ao $\mathrm{PE}$ nas décadas seguintes, houve a redução para cinco etapas, além da alteração na denominação de algumas delas. Como exemplo, a primeira etapa é também conhecida como coleta de $\operatorname{dados}^{(5,6)}$, termo adotado neste estudo.

Diante deste contexto, acredita-se a construção de um instrumento de coleta de dados estruturado com base no referencial teórico das NHB permite a avaliação do paciente como um todo indivisível no seu universo bio-psico-sócio-espiritual, facilitando o levantamento de dados/informações que auxi- liam o enfermeiro a determinar os Diagnósticos de Enfermagem e as intervenções individualizadas ${ }^{(5,6)}$.

Cabe ressaltar que existem três correntes de pensamentos em relação à terminologia utilizada na enfermagem: a primeira corrente considera os termos Processo de Enfermagem (PE), Metodologia da Assistência de Enfermagem (MAE) e Sistematização da Assistência de Enfermagem (SAE) com significados diferentes. A segunda corrente trata Metodologia da Assistência de Enfermagem e Processo de Enfermagem como sendo termos equivalentes. E a terceira corrente conclui que os três termos são sinônimos ${ }^{(7)}$. Os autores da primeira corrente de pensamento consideram os conceitos distintos porque a "sistematização da assistência demanda alguma ferramenta a ser utilizada e as ferramentas para sistematização demandam métodos de trabalho, previstos nas metodologias para a assistência de enfermagem. Enquanto que o processo de enfermagem é a metodologia da assistência de enfermagem proposta por cada uma das Teóricas de Enfermagem para o trabalho processual”(7).

A aplicação do PE coloca em evidência a assistência realizada, capaz de atestar e conferir a necessária cientificidade do fazer profissional e proporciona uma maior autonomia e visibilidade de suas ações, obtendo o real conhecimento da categoria $^{(8)}$, para isso, é imprescindível o registro de todas as atividades realizadas pelo enfermeiro ${ }^{(9)}$. Assim, destaca-se a necessidade e a relevância de um estudo que confira maior visibilidade da prática clínica da enfermagem e da participação de enfermeiros na construção de práticas de saúde pautadas no paradigma do cuidado integral e holista e contribua com a qualificação da assistência sistematizada e individualizada ao paciente crítico. Além disso, ressalta-se que essa problemática está relacionada à experiência profissional de uma das autoras como enfermeira de uma UTI.

Considerando o panorama apresentado, estabeleceu-se como objetivo deste estudo construir coletivamente um instrumento de coleta de dados para pacientes de Unidade de Terapia Intensiva, fundamentado na Teoria das Necessidades Humanas Básicas de Horta. Utilizou-se como ponto de partida para as discussões com enfermeiros um instrumento de coleta de dados existente na instituição.

\section{METODOLOGIA}

Trata-se de um recorte de uma Dissertação de Mestrado(10), que teve abordagem qualitativa 
e foi desenvolvida na Unidade de Terapia Intensiva (UTI) de um hospital de ensino de grande porte localizado na cidade de Porto Alegre, Rio Grande do Sul.

A coleta das informações ocorreu nos meses entre julho e outubro de 2009 por meio da técnica de Grupo Focal $(\mathrm{GF})^{(11)}$. Essa técnica vem sendo aplicada com freqüência nas pesquisas da área da saúde, principalmente na enfermagem, pois favorece a interação entre o pesquisador e os participantes com o objetivo de obter informações a partir da discussão focada em tópicos específicos e diretivos ${ }^{(12)}$.

Foram convidados a integrar a pesquisa um total de oito participantes: quatro enfermeiras assistenciais da Terapia Intensiva, uma de cada turno (M, T, NI e NII), sendo que duas eram preceptoras da Residência Integrada em Saúde (RIS) e as demais orientadoras, e quatro enfermeiras residentes do segundo ano do programa da RIS da ênfase em Terapia Intensiva. As enfermeiras residentes foram inseridas no Grupo Focal por fazerem parte de um processo de formação em serviço e com base na premissa de que sua inclusão enriqueceria as discussões em torno do tema, possibilitando o surgimento de diferentes opiniões e percepções em cada momento compartilhado, um momento singular e importante, proporcionando crescimento e aprendizagem profissional entre todos os envolvidos.

As participantes foram todas do sexo feminino e com conhecimento em Terapia Intensiva e no Processo de Enfermagem, o que favoreceu o processo de investigação. A moderadora do grupo focal foi a própria pesquisadora mestranda, a qual buscou capacitação durante curso de Mestrado, e uma enfermeira da instituição com conhecimento do Processo de Enfermagem foi convidada e capacitada para atuar como observadora. Para organizar os encontros, elaborou-se uma agenda contendo a descrição das atividades. Como guia de temas foi utilizado o próprio instrumento da instituição.

No primeiro encontro, realizou-se a apresentação entre os participantes, moderadora e observadora. Feito isso, apresentaram-se os objetivos do estudo, assim como, a trajetória do Grupo de Estudo dos Diagnósticos de Enfermagem e Prescrição Informatizada (GEDEPI) do HNSC, e sua relação com este estudo. Também se procedeu a explicação da técnica do Grupo Focal, ao esclarecimento das questões éticas e assinatura do Termo de Consentimento Livre e Esclarecido, conforme a resolução 196/1996.
Nas sessões subseqüentes, realizava-se uma breve síntese da reunião anterior e depois se discutia previamente o referencial teórico para subsidiar as participantes nessa construção coletiva. Como ponto de partida, utilizou-se o instrumento do GEDEPI, no intuito de reestruturar a forma e conteúdo conforme a teoria das Necessidades Humanas Básicas proposta por Horta. Para tanto, discutiu-se o acréscimo, a exclusão, a substituição e/ou a manutenção dos itens por meio da consulta a livros sobre anamnese e exame físico. Assim, foi se construindo os grupos e subgrupos de necessidades, levando em consideração a experiência profissional de cada uma.

As discussões produzidas em cada sessão do grupo focal foram analisadas conforme o preconizado por Horta, ou seja, grupos e subgrupos das $\mathrm{NHB}^{(2)}$.

A pesquisa seguiu os aspectos éticos previstos na Resolução 196/1996 $6^{(13)}$, obteve parecer favorável do Comitê de Ética da instituição de referência (CEP/GHC 058/09 - FR 258781) e cada participante expressou sua anuência em participar da investigação por meio de assinatura no Termo de Consentimento Livre e Esclarecido e foram adotados pseudônimos para identificar suas falas para preservar a confidencialidade das enfermeiras pesquisadas.

\section{RESULTADOS E DISCUSSÕES}

O instrumento de coleta de dados foi estruturado em sete grupos, assim distribuídos: Identificação; História pregressa; História da doença atual; Avaliação geral; Avaliação das Necessidades Psicobiológicas; Avaliação das Necessidades Psicossociais e Avaliação Necessidades Psicoespirituais. O grupo da Avaliação das Necessidades Psicobiológicas está constituído por 11 subgrupos de necessidades: Regulação Neurológica; Oxigenação; Percepção dos Órgãos dos Sentidos; Regulação Cardiovascular; Alimentação e Eliminação Intestinal; Hidratação e Eliminação Urinária; Integridade Física; Segurança Física; Sono e Repouso; Cuidado Corporal e Sexualidade. O grupo de Avaliação das Necessidades Psicossociais está composto por três subgrupos de necessidades: Comunicação, Gregária e Segurança Emocional. E o grupo de Avaliação das Necessidades Psicoespirituais contém os subgrupos religião, religiosidade e espiritualidade. A seguir, alguns resultados extraídos do trabalho original.

No grupo da identificação, um dos itens que gerou maior discussão foi a escolha da denominação 
raça ou cor, em função da dificuldade na sua definição, pois, ao longo dos anos, ocorreram miscigenações que contribuíram para a descaracterização fenotípica dos indivíduos, tornando difícil identificar determinada raça para um paciente crítico, o qual, muitas vezes, está impossibilitado de informar esses dados por estar sedado e/ou confuso. Desse modo, optou-se por deixar o termo cor por ser mais prático, apesar de gerar um dado subjetivo também passível de enganos. Algumas falas revelam essas dificuldades:

\section{É tão banalizado que as pessoas não valorizam a sua origem (Rosa, S2).}

Acho que não é uma coisa banalizada e sim confusa, levando o profissional ignorar a raça e nacionalidade se interessando só pela patologia (Azaléia, S2).

Na literatura, muitos estudos utilizam a terminologia cor de pele ou raça como uma variável demográfica, podendo estar associando a determinas doenças como Hipertensão Arterial Sistêmica (HAS), doença renal crônica, HDL, LDL e colesterol total, sendo evidenciado a prevalência maior na cor branca ${ }^{(14)}$. Nesse contexto, torna-se fundamental o profissional preencher o item cor na identificação do indivíduo, uma vez que poderá associá-la a uma melhor prevenção e controle, pois a maioria dessas doenças é modificável, possibilitando melhorar a qualidade dos cuidados à saúde, assim como a qualidade de vida, principalmente nas intervenções visando à pós-alta.

Na história pregressa, em relação ao item tabagismo, foram acrescentados os quesitos quantidade de cigarros consumidos por dia e o tempo de uso do tabaco. As participantes concluíram relevante incluir esse item no instrumento, conforme as falas exemplificadoras a seguir:

\section{A quantidade é importante para ver se ele é tabagista pesado ou não (Rosa, S2).}

Eu acho interessante, pois um familiar teve um AVC, fumava 3 maços/dia e acabou com DPOC (Jasmim, S2).

Resultados de um estudo cubano sobre os fatores de mortalidade atribuída ao consumo do tabaco indicam que em primeiro lugar está o câncer de pulmão, seguido da DOPC, cardiopatia isquêmica e enfermidade cerebrovascular ${ }^{(15)}$. No contexto hospitalar, ainda se encontram pacientes fazendo uso do tabaco durante a internação, seja escondido no quarto ou circulando no pátio aos olhos dos profissionais. Nesses casos, geralmente ocorre apenas advertência verbal em relação a essa prática, por ser um local considerado livre do tabaco. Um dos fatores imprescindível para o abandono do fumo é o manejo de uma equipe multiprofissional especializada, incluindo a participação da família para auxiliar com essa mudança de hábito.

Todas as doenças que compõem a história pregressa já estão contempladas no cronograma de atividades da residência de enfermagem da ênfase em Terapia Intensiva, porém ainda é necessária uma maior articulação com o campo da prática, pois o entendimento da fisiopatologia das doenças é comum a qualquer profissional da saúde e cada núcleo do saber utilizará essas informações de acordo com as suas competências específicas. Nessa ótica, a enfermagem utiliza instrumentos metodológicos para o planejamento de suas atividades. Esse é um aspecto importante, pois o campo de terapia intensiva em estudo deve disponibilizar condições técnicas para o residente desenvolver atividades específicas e legais, do profissional enfermeiro e, para isso, a sensibilização e conscientização da importância da aplicação das etapas do PE de forma adequada representam uma válvula propulsora para mudanças de paradigmas.

A parte do exame físico passou a ser denominada avaliação geral, por opção dos participantes do grupo. A verificação dos sinais vitais é uns dos primeiros cuidados prestados quando o paciente é internado na UTI. Em relação à dor, as participantes consideraram pertinente manter esse item no instrumento com o objetivo de aprimorar a qualidade da assistência, uma vez que a Agência Americana de Pesquisa e Qualidade em Saúde Pública e a Sociedade Americana de Dor consideram a dor o $5^{\circ}$ sinal vital, devendo ser mensurado e registrado com o mesmo rigor que os demais parâmetros vitais ${ }^{(16)}$.

Considerar a dor como o $5^{\circ}$ sinal vital proporciona imediata intervenção como ocorre nas demais alterações dos sinais vitais e exige dos profissionais reavaliações subseqüentes. Essa avaliação sistemática deve fazer parte da educação em saúde de todos os envolvidos com a assistência, incluindo os enfermeiros residentes que serão os multiplicadores de melhores práticas de cuidado em saúde e enfermagem.

No subgrupo regulação neurológica, em relação à avaliação das pupilas, as participantes sugeriram a utilização dos itens isocóricas, anisocóricas, direita maior que a esquerda e vice-versa, fotorreagentes sim ou não, mióticas e midriáticas 
e um espaço destinado para anotar o tamanho do diâmetro pupilar. As falas, a seguir, ilustram a importância deste item no instrumento.

Sempre é pertinente avaliar para se ter um parâmetro, dependendo da lesão cerebral, a avaliação da pupila será o primeiro sinal, o problema mesmo é ficar anisocórica muito rápido (Dália, S3).

Sim, na hora da avaliação neurológica você vai dizer, bom ele está mais midriático do que quando eu olhei anteriormente (Dália, S3).

A avaliação das pupilas rotineiramente é fundamental para o acompanhamento de possíveis alterações, pois em pacientes graves as modificações do estado neurológico podem ocorrer de uma hora para outra, o que torna imprescindível a observação e o registro do diâmetro das pupilas, da forma e da reação à luz, assim como a comunicação imediata das alterações encontradas ${ }^{(17)}$. A utilização de escalas na prática clínica pode facilitar e auxiliar a equipe a realizar uma avaliação mais precisa das condições neurológicas do paciente, o que possibilita a detecção precoce de problemas e a instituição de medidas para prevenir possíveis complicações consideradas modificáveis.

A inclusão do sono e repouso na avaliação do paciente crítico foi considerada importante pelas participantes do estudo. Elas decidiram deixar uma questão aberta, ou seja, somente com o item observações seguido de linhas pontilhadas que possibilitem ao profissional descrever as particularidades de cada paciente a partir das informações fornecidas por ele e/ou sua família, como hábitos, características e problemas relacionados ao sono e repouso. Vários fatores contribuem negativamente na qualidade dessa necessidade na terapia intensiva, como os ruídos dos diversos alarmes, das conversas em tom elevado, o excesso de iluminação artificial e os extremos de temperatura ${ }^{(18)}$.

Assim, a presença desse subgrupo no instrumento de coleta de dados possibilita ao enfermeiro a elaboração de intervenções de enfermagem que contribuam para melhoria da quantidade e qualidade do sono do paciente crítico e minimizem os efeitos dos fatores externos como os supracitados, pois todo e qualquer paciente deve ser tratado com respeito e dignidade. A conscientização dessas atitudes pelos profissionais da saúde envolvidos vai ao encontro de uma assistência mais humanizada, contribuindo para melhores respostas dos pacientes aos investimentos terapêuticos.
Nas Necessidades Psicossociais, as participantes consideraram relevante escolher somente as que são mais freqüentes na UTI. Como o subgrupo comunicação com o objetivo de identificar as condições da mesma pelo paciente, além do grau de entendimento do idioma português. No subgrupo gregária, procurar-se-á investigar se o paciente vive com a família ou com outra pessoa e no subgrupo segurança emocional será investigado em relação a sentimentos de ansiedade e medo, contendo um espaço para registrar o motivo dos mesmos. A implementação de medidas para amenizar os agravos à saúde por meio da comunicação terapêutica é indispensável na inter-relação dos indivíduos envolvidos no cuidado assistencial, pois proporcionam a detecção precoce de necessidades, anseios e conflitos do paciente e de seu familiar, desmistificando o próprio ambiente em que estão inseridos.

No ambiente da Terapia Intensiva, a busca da religião e da espiritualidade são fontes de energia para o enfrentamento da doença, proporcionando conforto, encorajamento e esperança para os envolvidos no processo saúde-doença. As participantes definiram a inclusão dessas necessidades na construção do instrumento, embora ainda pouco presente no cotidiano do enfermeiro. Ela contempla a investigação sobre crenças e religião do paciente, se é praticante ou não, e se gostaria de apoio religioso ou espiritual.

A enfermagem tem um importante papel de facilitadora e mediadora para que o paciente/família contemple suas necessidades espirituais, que foram afetadas com a mudança do estilo de vida. Esse cuidado implica dedicação, paciência, fazendo-se presente com disposição para ouvir e respeitar as crenças e os valores desses indivíduos ${ }^{(19)}$. Assim sendo, esse tema também precisa ser abordado em aulas teóricas e no campo das práticas do enfermeiro em formação, pois a essência do cuidado de enfermagem é o ser humano na perspectiva do paradigma holístico, ou seja, a compreensão do homem como um ser bio-psico-socioespiritual ${ }^{(20)}$.

Durante as discussões foram sugeridas, em cada sessão, o acréscimo, exclusão, substituição e/ou manutenção de itens. Alguns subgrupos ficaram somente com o item observação e linhas em branco para a descrição de alguma particularidade. As participantes argumentaram que um instrumento contendo informações que nem sempre serão utilizadas pode gerar um sentimento de frustração no profissional que está preenchendo, pois dificilmente conseguirá completá-lo. 
Após a formulação da versão final, o instrumento foi apresentado aos componentes do GEDEPI do HNSC, que o consideraram mais objetivo e completo, bem como apoiado em um referencial teórico, das NHB. Dessa forma, o grupo acordou que este instrumento será utilizado como padrão nas áreas em que o PE for implantado.

$\mathrm{Na}$ fase de testagem do instrumento de coleta de dados na UTI, algumas participantes encontraram dificuldade em conciliar o momento da internação do paciente com a sua disponibilidade para aplicação do instrumento devido à distância entre os campos de atuação dos residentes. A aplicação ocorreu em 10 pacientes, possibilitando que as participantes do estudo identificassem a ausência de itens não sugeridos durante a sua elaboração, sendo possível acrescentar, excluir e/ou substituir alguns itens para o refinamento do mesmo. Durante a testagem, surgiram algumas dúvidas em relação à interpretação de itens, desta forma, o grupo percebeu a necessidade de ter um manual de orientação para otimizar o trabalho do enfermeiro.

Como contribuição para o estudo, foi elaborado um manual de orientação para facilitar o registro do instrumento de coleta de dados. Este manual foi submetido à apreciação das integrantes do Grupo Focal, por meio eletrônico. O retorno dessa avaliação foi positivo, tanto em relação ao conteúdo quanto à apresentação visual. O manual possui algumas vantagens, como evitar interpretações ambíguas dos itens, sistematizar a avaliação, organizar o trabalho do enfermeiro e oferecer informações para uma consulta breve, salientando-se o aspecto educativo que estes materiais proporcionam.

A coleta de informações torna-se a fase fundamental para o desenvolvimento do Processo de Enfermagem, pois se configura como o alicerce que sustentará as demais etapas. Portanto, o instrumento de coleta de dados deve estar em consonância com a filosofia do serviço e com o local onde será implementado, para que os cuidados de enfermagem sejam direcionados a atender as necessidades dos pacientes evidenciadas no decorrer desse processo.

\section{CONCLUSÃO}

O presente estudo alcançou seu objetivo com a construção coletiva da primeira etapa do Processo de Enfermagem para pacientes críticos, fundamentado no modelo das NHB de Wanda Horta. A técnica do grupo focal oportunizou trocas de informações, idéias, opiniões e experiências profissionais entre enfermeiras residentes e enfermeiras assistenciais as quais são preceptoras e orientadoras da Residência Integrada em Saúde. As discussões geraram a construção de um conhecimento coletivo e comprometido, estando sempre presente o respeito, a responsabilidade, a dedicação e o companheirismo.

O Histórico de Enfermagem, aqui denominado coleta de dados, baseado nas NHB, poderá contribuir para uma maior aproximação, melhor comunicação e relacionamento interpessoal entre enfermeiros, pacientes e familiares, e uma assistência individualizada focada nas necessidades prioritárias dos pacientes com vistas a alcançar os resultados esperados. Essa etapa, estando bem estruturada, fornecerá sustentação para as demais, facilitando aos enfermeiros assistenciais e enfermeiros residentes o ensino da coleta de dados, bem como o desenvolvimento do pensamento crítico e raciocínio clínico na elaboração dos Diagnósticos de Enfermagem e no estabelecimento de intervenções de enfermagem adequadas e específicas para determinada situação. O registro correto dessas etapas pode ser utilizado para subsidiar futuras pesquisas, principalmente na residência, que tem a pesquisa como um quesito para conclusão de curso.

A utilização do PE na formação em serviço dos enfermeiros residentes, em consonância com os princípios da Educação Permanente, pode ser uma estratégia metodológica que possibilitará o desenvolvimento de um pensar problematizador, com a construção de novos conhecimentos a partir da realidade vivenciada. Sua aplicação nas instituições de ensino pode favorecer a formação de profissionais cientes das suas responsabilidades assistenciais e educacionais, fornecendo-lhes subsídios para atuarem como multiplicadores de melhores práticas de cuidado em saúde e enfermagem.

Sugere-se, para pesquisas futuras, a validação do instrumento de coleta de dados e do manual de orientação para o seu preenchimento a fim de verificar se os itens contemplados no mesmo possibilitam a identificação de sinais, sintomas e fatores de risco que embasem a elaboração dos DE prioritários em pacientes críticos. Recomenda-se, ainda, que Residências Integradas em Saúde incluam o ensino do Processo de Enfermagem na programação das atividades do residente enfermeiro, em consonância com a implementação desta metodologia nos campos de prática clínica. 


\section{REFERÊNCIAS}

1 Brandalize DL, Kalinowiski CE. Processo de Enfermagem: vivência na implantação da fase de diagnóstico. Cogitare Enferm. 2005;10(3):53-7.

2 Horta WA. Processo de enfermagem. São Paulo: EPU; 1979.

3 Neves RS. Sistematização da Assistência de Enfermagem em Unidade de Reabilitação segundo o Modelo Conceitual de Horta. Rev Bras Enferm. 2006;59(4):556-9.

4 Marques DKA, Moreira GAC, Nóbrega MML. Análise da teoria das necessidades humanas básicas de Horta. Rev Enferm UFPE On Line. 2008;2(4):410-16.

5 Galdeano LE, Rossi LA. Construção e validação de instrumentos de coleta de dados para o período perioperatório de cirurgia cardíaca. Rev Latino-Am Enferm. 2002;10(6):800-04.

6 Lima LR, Stival MM, Oliveira CR, Chianca TCM. Proposta de instrumento para coleta de dados de enfermagem em uma Unidade de Terapia Intensiva fundamentado em horta. Rev Eletrônica Enferm. [Internet] 2006 [citado 2009 mar 04];8(3):349-57. Disponível em: http://www.fen.ufg.br/revista/v9/n3/v9n3a05.htm

7 Fuly PSC, Leite, JL, Lima, SBS. Correntes de pensamento nacionais sobre sistematização da assistência de Enfermagem. Rev Bras Enferm. 2008;61(6):883-87.

8 Pivoto FL. Proposta de processo de enfermagem em Unidade de Tratamento Intensivo pós-operatória cardiológica [dissertação]. Rio Grande (RS): Programa de Pós-Graduação em Enfermagem, Universidade Federal de Rio Grande; 2008.

9 Brasil. Resolução COFEN no 358/2009, de 15 de outubro de 2009. Dispõe sobre a Sistematização da Assistência de Enfermagem e a implementação do Processo de Enfermagem em ambientes, públicos ou privados, em que ocorre o cuidado profissional de Enfermagem, e dá outras providências [Internet]. 2009 [citado 2009 dez 14]. Disponível em: www.portalcofen.gov. br/2007/downloads/RESOLUCAO3582009.doc.

\footnotetext{
Endereço do autor / Dirección del autor / Author's address:

Rosaura Costa Bordinhão

Av. Engenheiro Ludolfo Boehl, 1179, casa 46, Teresópolis

91720-150, Porto Alegre, RS

E-mail: rosaura.bordinhao@terra.com.br
}

10 Bordinhão RC. Processo de enfermagem em uma unidade de tratamento intensivo à luz da Teoria das necessidades humanas básicas [dissertação]. Porto Alegre: Escola de Enfermagem, Universidade Federal do Rio Grande do Sul; 2010.

11 Debus M. Manual para excelência en la investigación mediante grupos focales. Washington: Academy for Educational Development; 1997.

12 Ressel LB, Beck CLC, Gualda DMR, Hoffmann IC, Silva RM, Sehnen GD. O uso do Grupo Focal em pesquisa qualitativa. Texto Contexto Enferm. 2008; $17(4): 779-86$.

13 Brasil. Ministério da Saúde. Conselho Nacional de Saúde. Resolução nº 196, de 10 de outubro de 1996. Dispõe sobre as diretrizes e as normas regulamentadoras de pesquisa envolvendo seres humanos. Brasília; 1996.

14 Paganin A, Moraes MA, Pokorski S, Rabelo ER. Factors that inhabit the use of nursing language. Int J Nurs Terminol Classif. 2008;19(4): 150-7.

15 Pérez PV, Travieso TH, Roche RGG, Gorbea MB, Pérez TR, Fernández SJV. Mortalidad atribuible al tabaquismo em Cuba. Rev. Cub. Salud Pública 2009;35(2):1-13. 92

16 Sousa FAEF. Dor: o quinto sinal vital. Rev Latino Am Enferm. 2002;10(3):446-7.

17 Diccini S, Whitaker IY. Exame neurológico. In.: Barros ALBL, organizador. Anamnese e exame físico. Porto Alegre: Artmed; 2002. p.96-111.

18 Moreira ML, Castro ME. Percepção dos pacientes em Unidade de Terapia Intensiva frente à internação. Rev RENE. 2006;7(1):75-83.

19 Paula ES, Nascimento LC, Rocha SMM. Religião e espiritualidade: experiências de famílias de crianças com insuficiência renal crônica. Rev Bras Enferm.2009; 62(1):100-6.

20 Pedrão RB, Beresin R. O enfermeiro frente à questão da espiritualidade. Einsten (São Paulo). 2010; 8(1):85-9. 